\title{
Observed geomagnetic induction effect on Dst-related magnetic observations under different disturbance intensities of the magnetospheric ring current
}

\author{
Dan Xu*, Huaran Chen and Mengtan Gao
}

\begin{abstract}
Based on the spherical harmonic expansion of geomagnetic disturbance observed on the mid-latitude surface of the Earth, external and internal field separation is conducted in which the external component is magnetic disturbance caused by the magnetospheric ring current and the internal component is that raised by the correspondingly induced currents within the Earth. The objectives are to evaluate the influences of the induced internal field on the surface magnetic observations and to reveal the response performance of internal geomagnetic induction under different strengths of magnetospheric ring current fluctuations for better understanding of the disturbance storm time (Dst) index variations. The results show that the ratio of the internal component to surface observation does not remain constant in storm time. During the main phase of the storm, the ratio variation follows the pattern of logarithmic growth with storm evolution up to the top value at the Dst-minimum; then, the ratio slowly decreases in the long recovery phase. Multiple small logarithmic growths are superimposed on the traces of internal ratios, corresponding to temporary ring current intensification during the storm main phase and amplifying the effect of this intensification on surface magnetic observations. With the intensification of magnetospheric storms from the level of $(-200 n T,-100 n T)$ to $(-300 n T,-200 n T)$ and $(-500 n T,-300 n T)$ classified with the Dst-minimum, the top value of the ratio averaged for each storm group in the superposed epoch analysis method increases from the value of $0.295 \pm 0.014$ to $0.300 \pm 0.016$ and $0.308 \pm 0.015$, respectively. It is demonstrated that the geomagnetic induction exceeds the linear relation with the intensification of the external field, which is physically reasonable and coincident with the Faraday's law of induction. Due to the effects of high induction of the oceans and lateral heterogeneity of electric conductivity distribution in the upper mantle of the Earth, the geomagnetic induction and its contribution to surface geomagnetic disturbance vary significantly among observatories. This factor should be considered in the research of magnetospheric current systems.
\end{abstract}

Keywords: Geomagnetic storms; Geomagnetic induction; Dst index

\section{Background}

Geomagnetic variations on the Earth's surface with time scales of seconds to a year primarily result from dynamic processes in the ionosphere, magnetosphere, and heliosphere. Geomagnetic storms, the most severe magnetospheric disturbances, are caused by intensified energy and momentum injection when the magnetosphere encounters fluctuated solar wind structures such as coronal mass ejections (CMEs). The representative feature

\footnotetext{
* Correspondence: danxu@outlook.com

Institute of Geophysics, China Earthquake Administration, Beijing 100081, China
}

of such storms is a significantly intensified equatorial ring current encircling the Earth westward, which is caused mostly by the drifting of energetic particles filling the inner magnetosphere from 2 to $9 R_{E}$. The ring current-induced magnetic field is opposite the Earth's main field at the equator and leads to remarkable depression of magnetic observation during storm time (Gonzalez et al. 1994). The symmetric part of geomagnetic disturbance on the low-latitude surface is tracked and quantified by the disturbance storm time (Dst) index (Sugiura 1964) derived from variations of the horizontal components of geomagnetic observations at four low-

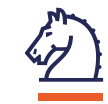


latitude geomagnetic observatories (Sugiura and Kamei 1991). The Dst variation is conventionally interpreted as a proxy of the symmetric ring current.

Recent research has indicated that geomagnetic disturbances due to the estimated ring current derived from both in situ magnetospheric particle measurements and simulated magnetospheric particle distributions have all underestimated Dst variation (e.g., Hamilton et al. 1988; Turner et al. 2001; Liemohn and Jazowski 2008). In fact, temporal variation of the magnetospheric ring current can excite induced currents in the Earth, which give rise to the secondary magnetic field and lead to further magnetic disturbances observed on the Earth's surface. As a result, the observed surface magnetic disturbances contain a contribution of the induced magnetic field originating from internal regions of the Earth responding to time variation of the magnetospheric ring current.

The induced magnetic disturbances from the Earth's interior sensed by geomagnetic observatories have been utilized to reveal mantle electrical conductivity structures since this method was implemented (e.g., Banks 1969; Schultz and Larsen 1990; Constable 1993; Olsen 1999; Kelbert et al. 2009; Khan et al. 2011). This technique has been valued as the most important method for studying mantle conductivity. Even for geomagnetic satellites orbiting at an altitude of $450 \mathrm{~km}$, the induced magnetic field still can be detected and used for mantle studies (Constable and Constable 2004; Martinec and McCreadie 2004; Kuvshinov and Olsen 2006; Püthe 2013a, b; Velímský 2013). For convenience, the magnetospheric currents are referred to as external sources, and the correspondingly induced currents and magnetic fields within the Earth are referred to as internal sources with respect to the magnetic field observed on the surface of the Earth.

The spatial configurations and fluctuating periods of external source currents in the magnetosphere and ionosphere, the conductivity distribution features inside the Earth, and the locations of magnetic probes are all key elements for the measurement of induction effects within the Earth (Mareschal 1986). The internal proportion could account for one-third of the magnetic observation in an idealized situation such that the Earth is perfectly conducting and the involved order of the spherical harmonic for external current configuration is only $n=1$ (Price 1967). Häkkinen et al. (2002) examined magnetic observations from $D s t$ standard observatories for 12 storms ranging from -76 to $-216 \mathrm{nT}$ in 1997 and 1998; the average internal contribution to Dst at roughly $30 \%$ during the storm main phase decreased to about $20 \%$ during the recovery phase. For the actual magnetic observations, the internal contribution is less than that in the idealized case, but it is still remarkable and deserves careful investigation.
From the condition of Dst variation, the magnetospheric disturbance and the ultimate source in solar wind can be approximately evaluated through analysis of works that differentiate solar wind disturbance structures and identify the solar wind sources of geomagnetic storms with different intensities (Gonzalez et al. 2011). Since the Dst index can be conveniently obtained from geomagnetic observatories, Dst variation is the most common and efficient indication of magnetospheric disturbances, particularly for geomagnetic storms. Extreme geomagnetic storms can adversely affect the instruments of satellites in the inner magnetosphere and surface power systems; therefore, Dst index prediction is essential for satellite exploration and daily human life. Solar wind plasma and interplanetary magnetic field measurements ahead of the Earth's bow shock are commonly used as basic input parameters and are sometimes the only input parameters (e.g., Temerin and Li 2002, 2006) used to predict $D s t$ variations. These predictions are mostly acceptable if the departure is ignored to some extent because solar wind disturbances are the most important driving sources for magnetospheric disturbance. However, accuracy in space physics and Dst prediction in fact requires determination of the exact contribution of internal induced current to Dst depression with different levels. Therefore, the objective of this study is to determine whether any differences in internal contribution to the Dst index occur in geomagnetic storms with different intensities.

\section{Methods}

\section{Dst calculation}

Dst is an hourly index calculated from the horizontal component of four geomagnetic observatories at middle latitudes: Honolulu ( $\mathrm{HON}$, geomagnetic colatitude and longitude: $68.45^{\circ}, 270.73^{\circ}$ ), San Juan (SJG, $62.31^{\circ}, 6.87^{\circ}$ ), Hermanus (HER, $123.99^{\circ}, 85.14^{\circ}$ ), and Kakioka (KAK, $62.31^{\circ}, 209.58^{\circ}$ ), which are located with sufficient difference from both auroral and equatorial electrojets and are evenly distributed in longitude (Sugiura and Kamei 1991).

In this paper, we adopt the basic Dst calculation method of Sugiura and Kamei (1991) following Häkkinen et al. (2002) to acquire the disturbed magnetic component by eliminating the baseline of secular variation due to the Earth's main magnetic field and its long period variation, crustal magnetic field, and the field from stable external current systems at the quiet time of the magnetosphere and ionosphere. We then subtract the solar quiet daily variation $S_{q}$. The remnant is considered to be a combination of fields resulting from disturbed magnetospheric current systems as well as the correspondingly induced currents in the Earth, referred to for convenience in this paper as Dst-related magnetic disturbances. 
The baseline of each magnetic component is computed by first calculating the annual mean value with the five quietest days of each month in the considered year and the preceding years. Then, we conducted a second-order polynomial fit to the annual mean values to obtain the baseline for each day.

$$
B_{x, y, z}^{\mathrm{base}}=a_{x, y, z}+b_{x, y, z} T+c_{x, y, z} T^{2}
$$

This data process procedure is conducted for all three components of surface magnetic observations, $B_{x}, B_{y}$ and $B_{z}$, in local Cartesian coordinates. In the standard Dst derivation method, only the horizontal component of the field is considered. However, because the $B_{z}$ component is indispensable for studying the interior electric structures of the Earth, the radial component is equally processed in our research following the same procedure as that used for the horizontal component.

Sugiura and Kamei (1991) expanded the $S_{q}$ as a double Fourier series in local time $T$ and month number $M$ to statistically fit $S_{q}$ curves for each day in 1 year.

$$
S_{q}(T, M)=\sum_{m=1}^{6} \sum_{n=1}^{6} A_{m n} \cos \left(m T+\alpha_{m}\right) \cos \left(n M+\beta_{n}\right)
$$

In equation (2), we take the same maximum value for $m$ and $n$ as that reported by Häkkinen et al. (2002). The coefficients $A_{m n}, \alpha_{m}$, and $\beta_{n}$ are determined by computing one $S_{q}$ curve for each month with the average of the five quietest days in the month.

The disturbed field accounting for Dst variations at each observatory is then obtained from the equation $B_{x, y, z}=B_{x, y, z}^{\text {obs }}-B_{x, y, z}^{\text {base }}-S_{q}(T)$. Then, the Dst index is derived from the four standard stations through the formula:

$$
\operatorname{Dst}(T)=\sum_{n=1}^{4} \frac{H_{n}(T)}{\cos \vartheta_{n}},
$$

where $H$ stands for the disturbed horizontal field and $\vartheta$ is the geomagnetic latitude at each station.

\section{Separation of internal and external fields}

The Dst-related magnetospheric ring current possesses the predominant $P_{1}^{0}$ spherical harmonic geometry in midlatitude regions (Banks 1969). Moreover, the Dst-related magnetic disturbances can provide an estimation of internal and external coefficients of Dst variations through spherical harmonic expansion of the disturbed field. Due to the consideration of conciseness in the physical concept, the reference frame of the disturbed field is converted from a geographic to geomagnetic coordinate system on the surface of the Earth.

The region between the Earth's surface and the ionosphere is generally current source-free, in which the magnetic field is conservative or curl-free and can thus be expressed as the gradient of a scalar potential (Lorrain and Corson 1970; Jackson 1998; Backus et al. 1996; Sabaka et al. 2010), known as potential magnetic field, as shown in equation (4).

$$
\vec{B}=-\nabla V
$$

Additionally, based on Maxwellian equations, the magnetic field vector must be solenoid, which implies:

$$
\nabla^{2} V=0
$$

The potential $V$ is harmonic, and this Laplace equation can be solved easily in a geomagnetic spherical coordinate system $(r, \theta, \varnothing)$ where $r$ is the radial distance from the center of the Earth, $\theta$ is the geomagnetic colatitude angle ringing from $0^{\circ}$ at geomagnetic north pole to $180^{\circ}$ at the south pole, and $\varnothing$ is the longitude angle expressed as a spherical harmonic expansion of associated Legendre polynomials $P_{l}^{m}$, with Schmidt quasi-normalized spherical harmonic coefficients $\left(i_{l}^{m} e_{l}^{m}\right)$ representing the internal and external magnetic fields:

$$
V(r, \theta, \phi)=a \sum_{l=1}^{\infty} \sum_{m=-1}^{l}\left\{i_{l}^{m}(t)\left(\frac{a}{r}\right)^{l+1}+e_{l}^{m}(t)\left(\frac{r}{a}\right)^{l}\right\} p_{l}^{m}(\cos \theta) e^{i m \phi}
$$

where $a$ is the Earth's radius. Keeping only the $P_{1}^{0}$ contribution for Dst-related disturbances under the assumption of symmetrical ring current (Constable and Constable 2004; Kuvshinov and Olsen 2006),

$$
V(r, \theta)=a\left\{i_{1}^{0}(t)\left(\frac{a}{r}\right)^{2}+e_{1}^{0}(t)\left(\frac{r}{a}\right)\right\} P_{1}^{0}(\cos \theta) .
$$

By using equation (7), the magnetic field vector $\rightarrow B$ of disturbed field as components $B_{n} B_{\theta}$, and $B_{\varnothing}$ in the geomagnetic spherical coordinate system and thus the symmetric geomagnetic disturbance can be expressed as:

$$
\begin{gathered}
B_{r}=\left[-e_{1}^{0}+2 i_{1}^{0}\left(\frac{a}{r}\right)^{3}\right] \cos \theta \\
B_{\theta}=\left[e_{1}^{0}+i_{1}^{0}\left(\frac{a}{r}\right)^{3}\right] \sin \theta \\
B_{\phi}=0
\end{gathered}
$$

The $B_{\theta}$ component represents the southpole-directed horizontal component of Dst-related magnetic field disturbance in the geomagnetic spherical coordinate system, the $B_{r}$ component is the same as the disturbed $B_{\mathrm{z}}$ component with the opposite direction, and the azimuthal magnetic component $B_{\varnothing}$ is zero for the symmetrically distributed magnetospheric ring current. For the observations from geomagnetic observatories, $r=a$ 
approximately. Then, the external $e_{1}^{0}$ and internal $i_{1}^{0}$ coefficients can be resolved directly from equation (8).

$$
\begin{aligned}
e_{1}^{0} & =-\frac{1}{3}\left[\frac{B_{r}}{\cos \theta}-\frac{2 B_{\theta}}{\sin \theta}\right] \\
i_{1}^{0} & =\frac{1}{3}\left[\frac{B_{r}}{\cos \theta}+\frac{B_{\theta}}{\sin \theta}\right]
\end{aligned}
$$

Then, the contribution rates of the external field $\left(B_{e}\right)$ and internal field $\left(B_{i}\right)$ to the symmetrical disturbed field $\left(B_{\theta}\right)$ on the surface observatories can be expressed as:

$$
\begin{aligned}
& R_{i}=\frac{B_{i}}{B_{\theta}}=\frac{i_{1}^{0}}{i_{1}^{0}+e_{1}^{0}}=\frac{1}{3} \frac{B_{r}}{B_{\theta}} \tan \theta+\frac{1}{3}, \\
& R_{e}=\frac{B_{e}}{B_{\theta}}=\frac{e_{1}^{0}}{i_{1}^{0}+e_{1}^{0}}=\frac{2}{3}-\frac{1}{3} \frac{B_{r}}{B_{\theta}} \tan \theta .
\end{aligned}
$$

Then, the external contribution to the Dst index can be derived from the external part $B_{e}$ of each station and equation (3).

Assuming that the interior of the Earth is a perfect electric conductor, in which the fluctuated period of external field should be sufficiently fast and the interior conductivity within the Earth should be sufficiently large according to the penetration depth $\left(\delta=\sqrt{T / \pi \mu_{0} \sigma}\right)$ of the magnetic field, internal currents would be induced to prevent any external field from entering the conductor, and the radial magnetic component measured on the surface will vanish. This means that internal induced currents have excited magnetic fields in which radial component cancels the radial part of the external field. In equation (10), when the radial component of the magnetic field is zero, the internal part is one-third of the horizontal component, which coincides with the inference of Price (1967).

\section{Results and discussion}

We adopted the geomagnetic observations of 68 intense geomagnetic storms (Dst $\leq-100 n T)$ at four Dst geomagnetic observatories recorded from 2000 to 2013. The standard method for Dst index derivation reported by Sugiura and Kamei (1991) and the internal/external field separation technique introduced in the last section were utilized. The objectives were to investigate the observational performances of geomagnetic induction from internal induced currents of the Earth and the possible divergence of their contributions to Dst variations with different intensities to improve the accuracy in Dst prediction.
The selected storms were divided into three classes according to their intensities, as shown in equation (12) and Table 1.

$$
\begin{aligned}
& \text { G1 : Dst } \in(-200 n T,-100 n T] \\
& \text { G2 }: \text { Dst } \in(-300 n T,-200 n T] \\
& \text { G3 : Dst } \in(-500 n T,-300 n T]
\end{aligned}
$$

These storms literally belong to intense storms according to the classification of (Gonzalez et al. 1999), of which $80 \%$ could have been caused by coronal mass ejection (CME; Xu et al. 2009). For CME, the sheath region and the magnetic flux rope with a southward interplanetary magnetic field after high-speed interplanetary shock is the dominant source responsible for the presence of the main phase of intense storms, particularly with significant Dst depression. Consequently, the main phases of intense storms led by CMEs have an average duration of about $10 \mathrm{~h}$, which is obviously shorter than the average main-phase duration of about $27 \mathrm{~h}$ for weak storms led by interplanetary corotating interaction regions (CIRs; Hutchinson et al. 2011). For geomagnetic induction from internal induced currents, the variation period of external sources is a critical issue for determining the penetration depth under certain observatory conditions. Within this depth of interior layers, the actual heterogeneity of electric conductivity distribution is responsible for the discrepancy of electric and magnetic induction affecting surface observations; beyond this depth, the external magnetic signal is generally shielded. Moreover, for actual Earth conditions, the phase lag of the response relation between the induced internal and external source field depends on the varying periods of the external source field. However, different from intense storms, nearly $40 \%$ of storms with $D s t$ above $-100 \mathrm{nT}$ could be resulted by CIRs, thus possessing a much longer storm main phase. Therefore, only the data of intense storms were adopted in this research under the assumption that they all have gone through nearly the same time scale of main phase duration. In such cases, similar penetration depth at a certain observatory may be guaranteed for all storms.

The superposed epoch analysis (SEA) technique is effective for resolving signals from noise and is used for clearly determining and quantifying the response signal to forcing events under the assumption that the selection of forcing events is based on solid physical criteria. In this research, SEA was applied to reveal the response relation of geomagnetic induction from internal currents to different variation scales of external magnetospheric current systems at low-latitude regions of the Earth's surface. The SEA method adopted here used average durations of storm phases. The specifically chosen epochs for SEA were the main and recovery phases. The average durations were set as 15 and $36 \mathrm{~h}$ for the main phase 
Table 1 Adopted intense geomagnetic storms from 2000 to 2013

\begin{tabular}{|c|c|c|c|c|c|c|c|}
\hline \multicolumn{6}{|c|}{ Dst $\in(-200 \mathrm{nT},-100 \mathrm{nT}]$} & \multicolumn{2}{|c|}{ Dst $\in(-300 \mathrm{nT},-200 \mathrm{nT}]$} \\
\hline Date & $\begin{array}{l}\text { Dst mini. } \\
(\mathrm{nT})\end{array}$ & Date & $\begin{array}{l}\text { Dst mini. } \\
\text { (nT) }\end{array}$ & Date & $\begin{array}{l}\text { Dst mini. } \\
\text { (nT) }\end{array}$ & Date & $\begin{array}{l}\text { Dst mini. } \\
\text { (nT) }\end{array}$ \\
\hline 20000212 & -110 & 20020419 & -148 & 20050508 & -110 & 20000406 & -288 \\
\hline 20000524 & -147 & 20020511 & -110 & 20050530 & -113 & 20000812 & -235 \\
\hline 20001005 & -182 & 20020523 & -109 & 20050612 & -106 & 20000917 & -201 \\
\hline 20001014 & -107 & 20020904 & -109 & 20050824 & -142 & 20010411 & -236 \\
\hline 20001029 & -126 & 20020907 & -153 & 20050831 & -122 & 20011106 & -259 \\
\hline 20001106 & -159 & 20021001 & -176 & 20050911 & -127 & 20011124 & -221 \\
\hline 20001129 & -119 & 20021004 & -125 & 20061215 & -162 & 20041109 & -263 \\
\hline 20010320 & -149 & 20021121 & -128 & 20110806 & -107 & 20050515 & -247 \\
\hline 20010418 & -114 & 20030529 & -144 & 20110926 & -101 & \multirow{2}{*}{\multicolumn{2}{|c|}{ Dst $\in(-500 \mathrm{nT},-300 \mathrm{nT}]$}} \\
\hline 20010422 & -102 & 20030618 & -141 & 20111025 & -132 & & \\
\hline 20010817 & -105 & 20030711 & -105 & 20120309 & -118 & Date & Dst mini. \\
\hline 20010926 & -102 & 20030817 & -148 & 20120424 & -108 & & $(n T)$ \\
\hline 20011001 & -148 & 20040122 & -130 & 20120715 & -127 & 20000715 & -301 \\
\hline 20011003 & -166 & 20040403 & -117 & 20121001 & -119 & 20010331 & -387 \\
\hline 20011021 & -187 & 20040722 & -100 & 20121008 & -105 & 20031029 & -353 \\
\hline 20011028 & -157 & 20040724 & -136 & 20121114 & -101 & 20031030 & -383 \\
\hline 20020324 & -100 & 20040727 & -150 & 20130317 & -132 & 20031120 & -422 \\
\hline 20020417 & -127 & 20040830 & -129 & 20130601 & -119 & 20041107 & -374 \\
\hline
\end{tabular}

and the recovery phase, respectively, according to typical durations of intense storm developments. The data for each storm phase were shifted to fit these durations to superpose the epochs of all selected storm phases. The onset of the main phase and the Dst-minimum were the common reference times for this superposition. The onset of the main phase was chosen as the point followed by sudden drops in Dst value, and the Dst-minimum was the start of the recovery phase.

For each storm, the baseline of secular variation was removed from the original magnetic observation in each station. The field derived from the $S_{q}$ current system was subsequently eliminated from the remnant. Next, the residual field was considered as Dst-related magnetic variation originating from disturbed magnetospheric current systems and the induced currents in the Earth. These Dst-related disturbances were then projected to the geomagnetic spherical coordinate system on the Earth's surface $\left(B_{n} B_{\theta}, B_{\varnothing}\right)$ to conduct the separation procedure of internal and external components based on equation (9) for each storm at each station.

With the separated internal and external fields, the periods of the main and recovery phases of storms were extracted to obtain the mean values for each storm category by performing SEA. Figures 1, 2, 3, and 4 demonstrate the SEA results obtained at HON, KAK, SJG, and HER stations. The dashed lines in the upper panels show the storm time variations of internal part $B_{i}$ and external part $B_{e}$ out of the disturbed horizontal field $B_{\theta}$ of dipole symmetry; $B_{\theta}$ fields appear as solid lines. The bottom two ratio components illustrate the traces of field ratios $\left(R_{i}=B_{i} / B_{\theta}, R_{e}=B_{e} / B_{\theta}\right)$ over the period of storm evolution. Blue and green lines represent internal and external field ratios, respectively, as indicated by the graph legends. The ambient margins around the curves in the ratio panels illustrate the standard deviations of the SEA process, which measure the spread of datasets and the dispersion from the SEA mean values. In each panel, the horizontal axis measures time in hours, and the Dst-minimum is marked as the zero point demonstrated by the black vertical dotted line, which indicates the end of the storm main phase and the beginning of the recovery phase during the evolution of the geomagnetic storm. The start point of the time axis shows the onset of the storm main phase. The three columns in the graph show SEA results for G1, G2, and G3 storm levels, respectively.

Figure 1 presents the SEA mean values of magnetic fields and their ratios at $\mathrm{HON}$ station. According to the upper three panels of magnetic field components, it is obvious that internal and external fields followed the same variation pattern as that exhibited by the changes in the surface dipole symmetric field $B_{\theta}$ during the storm time, in which the fields significantly dropped from the onset point during the storm main phase until 


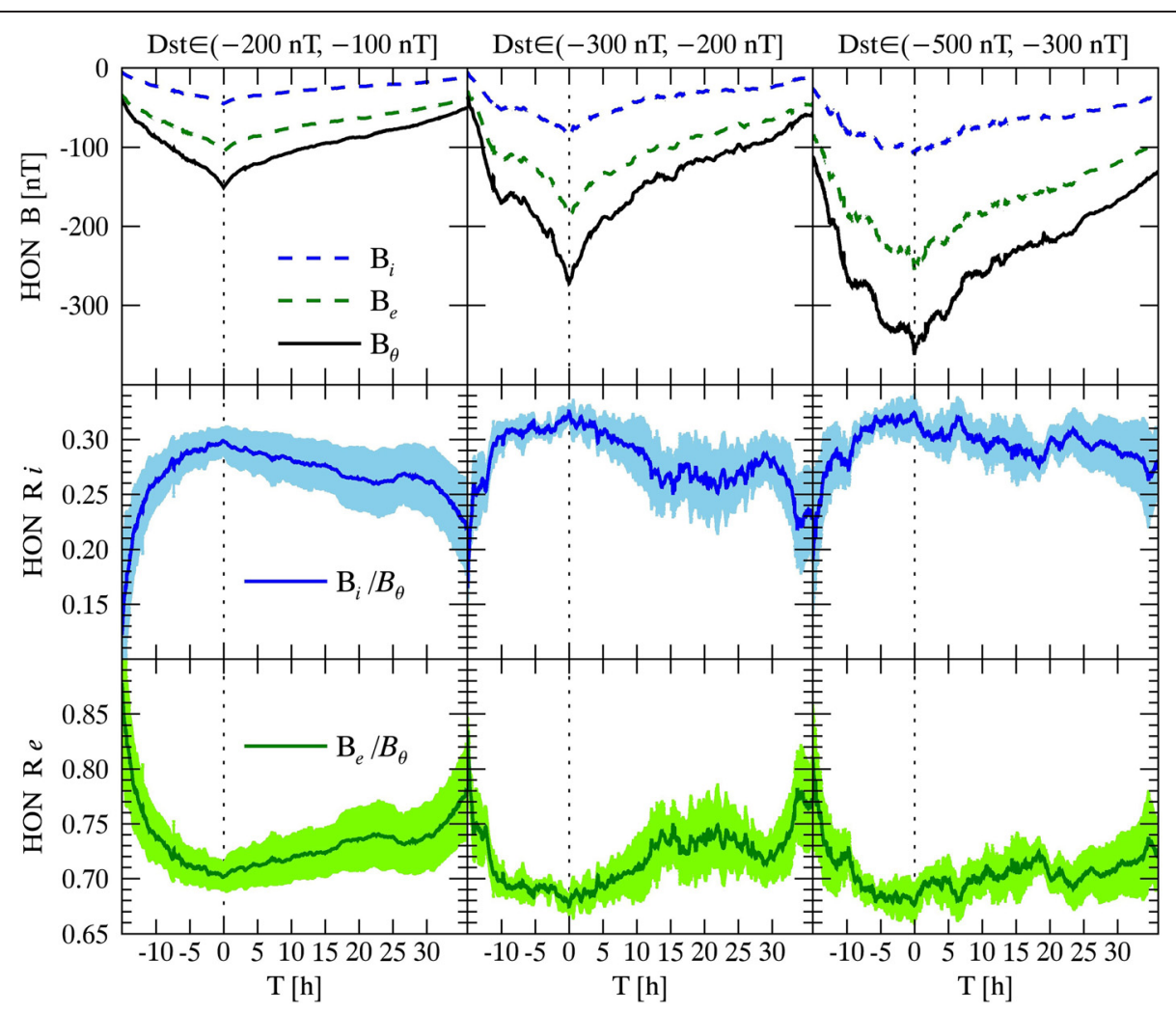

Figure 1 Superposed epoch analysis (SEA) results of magnetic components and the related ratios during the storm time at HON. The SEA mean values of the dipole symmetrical horizontal components $\left(B_{e}\right)$ of geomagnetic observations in geomagnetic spherical coordinate, external fields $\left(B_{e}\right)$ arising from the disturbance of the magnetospheric ring current and internal induced fields $\left(B_{i}\right)$, and the internal and external contributions $\left(R_{i}=B_{i} / B_{\theta}, R_{e}=B_{e} / B_{\theta}\right)$ are shown with their standard deviations.

achieving the minimum value and gradually recovering. For the G1 category in the left column of the graph, the internal ratio $R_{i}$ shows rapid growth in the duration of the main phase in a logarithmic manner and slowly decreases after reaching the top value at the point of the Dst-minimum. The standard deviation was smaller at the points around the Dst-minimum than regions before and after, suggesting that the SEA mean value estimation is statistically somewhat more reliable in the data points around the Dst-minimum. The external ratios $R_{e}$ were the complete reverse of the internal ratios. The field ratios illustrate that for the surface magnetic observations, the ratio of the external fluctuation part caused by the magnetic ring current decreased whereas the ratio of the internal induced field increased during the storm main phase, reaching extreme values at the Dst-minimum before achieving slow recovery. For the sake of simplicity, the physical parameters of the magnetic fields and ratios referred in the SEA results all represent their SEA mean values.

As the storm levels intensified, the traces of the $B_{\theta}$ field clearly showed multiple small intensifications during the main phases, with one obvious dip for the G2 category in the middle column and three dips for G3 in the right column of the graph. These small dips mainly resulted from multiple temporarily intensified injections of charged particles into the magnetic ring current or possible energization mechanisms of ring current particles during the storm main phase. Clearly, larger storms normally contain more temporarily intensified injections of charged particles. The internal external part of the magnetic field and their contribution ratios all showed evidence of intermittent intensification. Moreover, for each temporary intensification of the external field, the internal ratio experienced a sudden increase correspondingly, as shown in the lower panels of the graph. For each category, the maximum of the $R_{i}$ mean value was $0.298 \pm 0.012$ at the Dst-minimum for G1 and increased to $0.325 \pm 0.006$ and $0.324 \pm 0.015$ for G2 and G3, respectively. During the main phase of G2 and G3, the values of $R_{i}$ were remarkably larger than those of G1, even in the recovery phase.

At KAK and SJG stations, the variation trend of magnetic field components and their ratios nearly maintained the same values as $\mathrm{HON}$ for each category, although the significant temporary intensifications were lower than those of $\mathrm{HON}$ during the main phases. For the G1 category, the external field $B_{e}$ nearly maintained the same values among all $D s t$ stations, which somewhat proves the polar-axis symmetry of the magnetic ring 


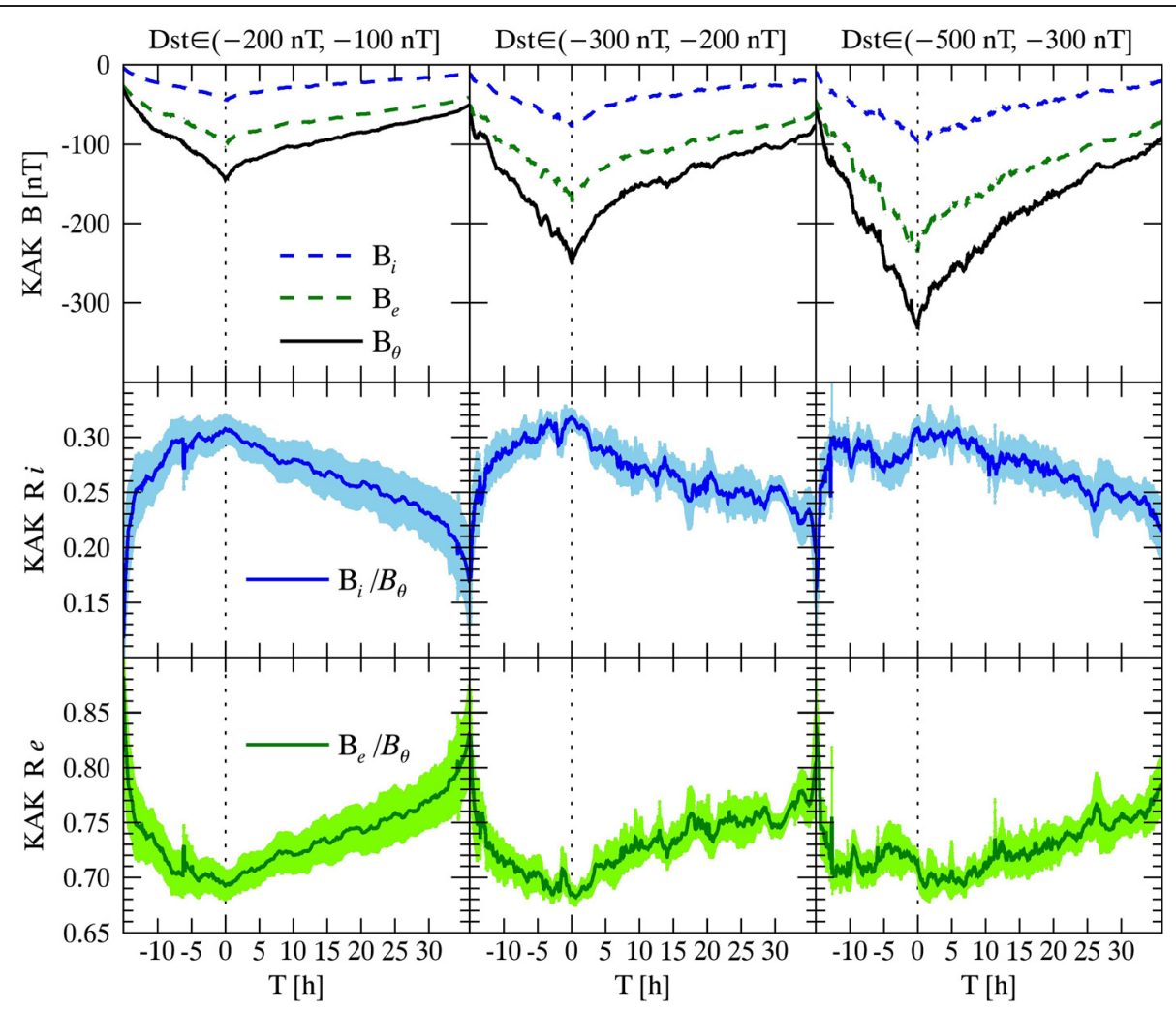

Figure 2 Superposed epoch analysis (SEA) results of magnetic components and the related ratios during the storm time at KAK.

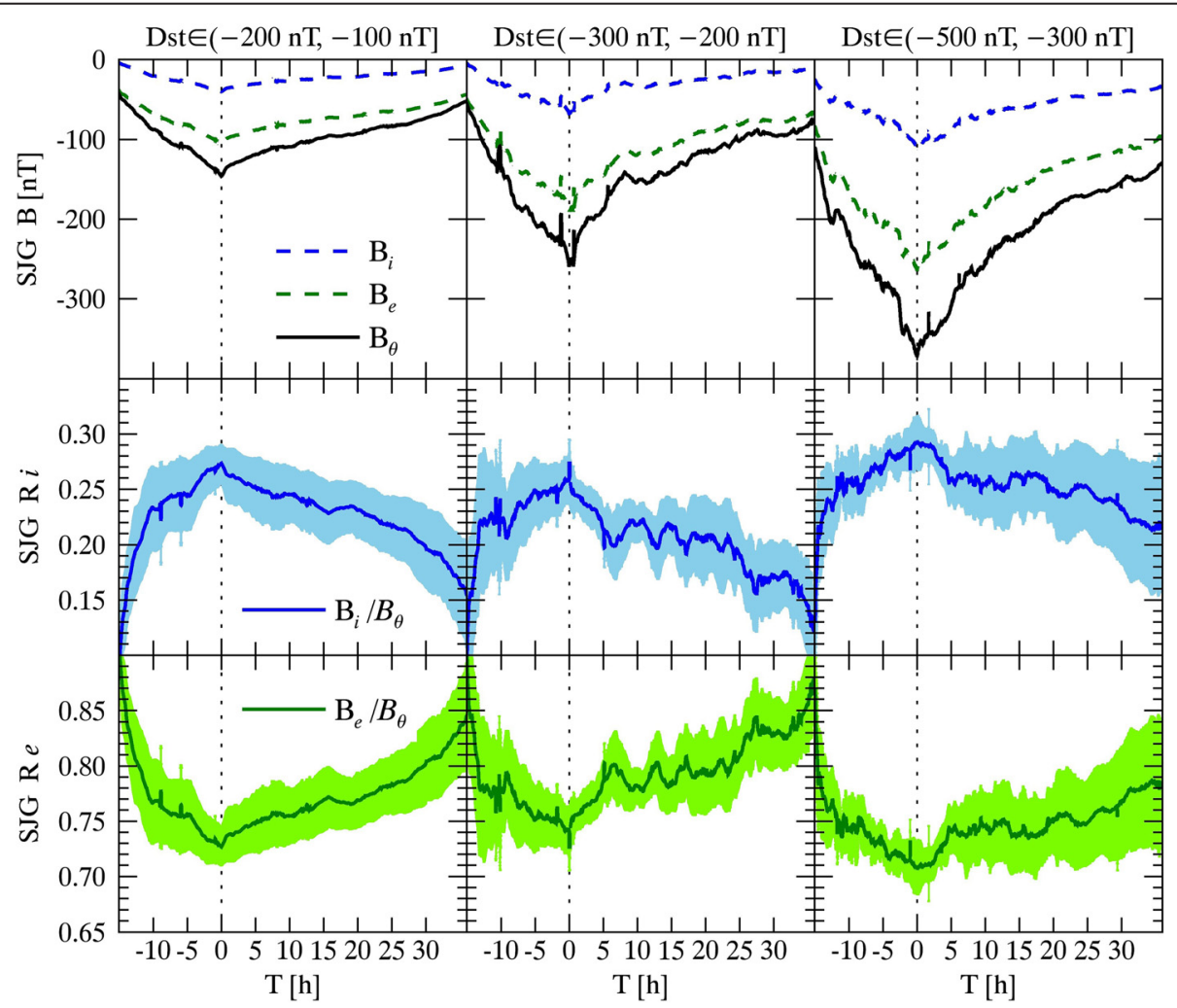

Figure 3 Superposed epoch analysis (SEA) results of magnetic components and the related ratios during the storm time at SJG. 


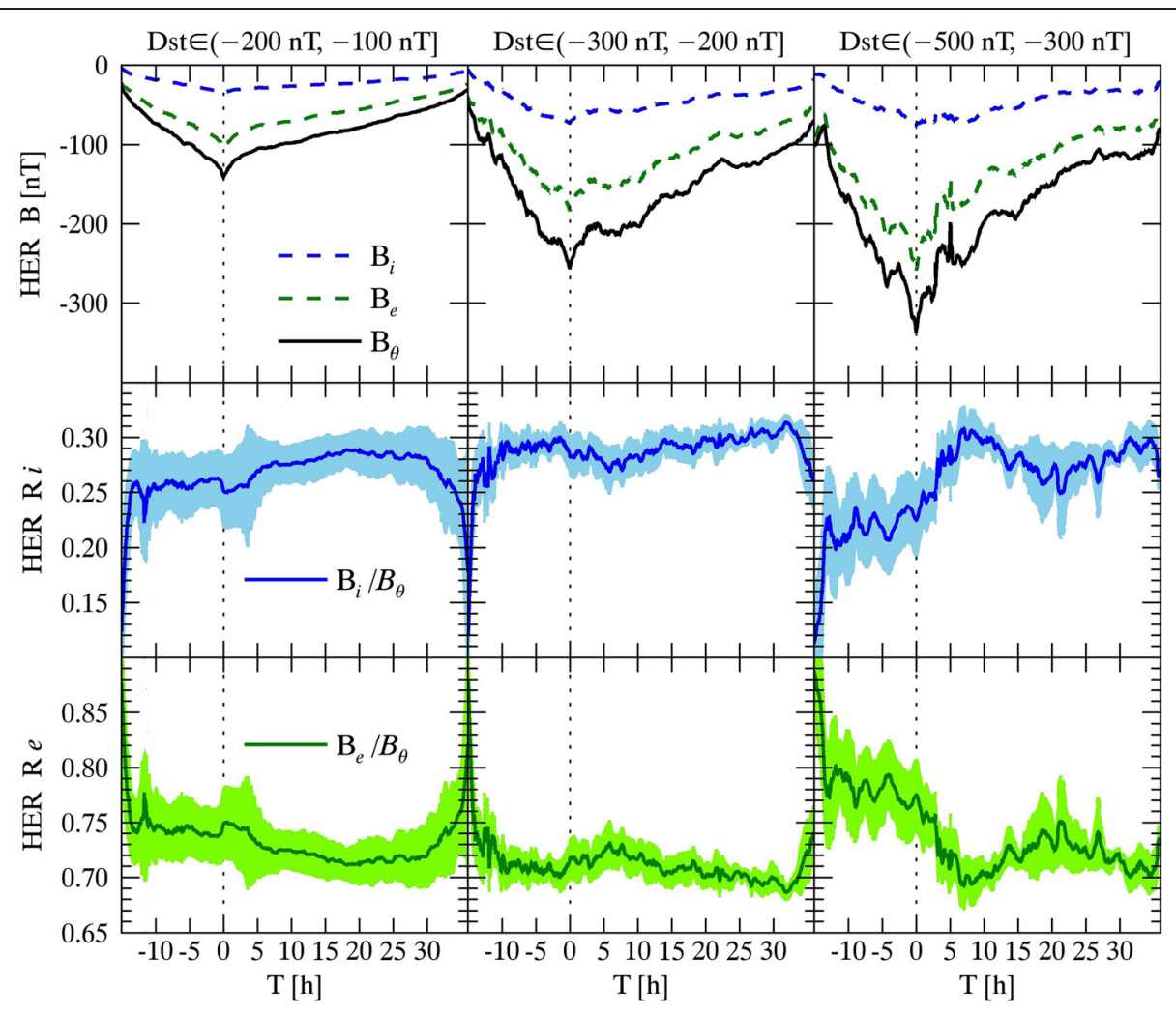

Figure 4 Superposed epoch analysis (SEA) results of magnetic components and the related ratios during the storm time at HER.

current under the storm level of the G1 category. For G2 and G3 categories, however, the $B_{e}$ values were slightly different. This difference may have been partly caused by the much less statistical cases in G2 and G3 categories and unavoidable missing data. At KAK station for G3, although the top value of $R_{i}(0.308 \pm 0.009)$ was just equal to that of the G1 category $(0.307 \pm 0.013)$, the value of $R_{i}$ was still larger than that G1 at the points during the main and recovery phases. At SJG station, the top value of $R_{i}$ was $0.273 \pm 0.017$ in G1 and above $0.292 \pm$ 0.023 in G3, showing an obvious increase. The $R_{i}$ values during the main and recovery phases in G3 were on average larger than those of G1 and G2.

For HER station, the tendency of $R_{i}$ variation remained the same for three storm groups. The values were unusually larger and reached the top value in the recovery phase, which differs in pattern from that of HON, KAK, and SJG. This situation of HER is similar to that reported by Häkkinen et al. (2002), in which the internal contribution is very small in the storm main phase but is larger and nearly remains constant in the recovery phase. The upper panel in Figure 5 shows the averaged value of the $B_{\theta}$ field and its internal and external parts over all selected storms in each station, and the lower panel illustrates the corresponding variations of the $R_{i}$ values. It is clear that the value of $R_{i}$ at KAK was slightly larger than that at HON during the main phase, Dstminimum, and early recovery phase but was obviously larger than that at SJG with a maximum divergence of nearly 0.04 . The situation at HER is different from other three stations, as previously mentioned. These systematic differences among stations are likely caused by differences in local conductivity structures, particularly the high induction of oceans near these observatories. Moreover, the lateral inhomogeneity of the underground conductivity structure of the Earth is likely responsible for the differences among the Dst stations.

For the selected intense storms, the characteristic duration of the main phase was about $10 \mathrm{~h}$. According to the penetration depth, the disturbed external magnetic field should penetrate through the asthenosphere into the mantle. This criterion should allow for strong attenuation in the highly conducting layer below the middle mantle of $700 \mathrm{~km}$. In the upper mantle region between 410 and $670 \mathrm{~km}$, the conductivity under KAK station is obviously larger than that under HON station, and the conductivity under SJG station is much less than that under both KAK and HON (Kelbert et al. 2009). Our results of internal contribution divergence among KAK, HON, and SJG stations are mostly coincident with the lateral inhomogeneity of conductivity distribution under these stations. Therefore, the remarkable lateral conductivity discrepancy in the 


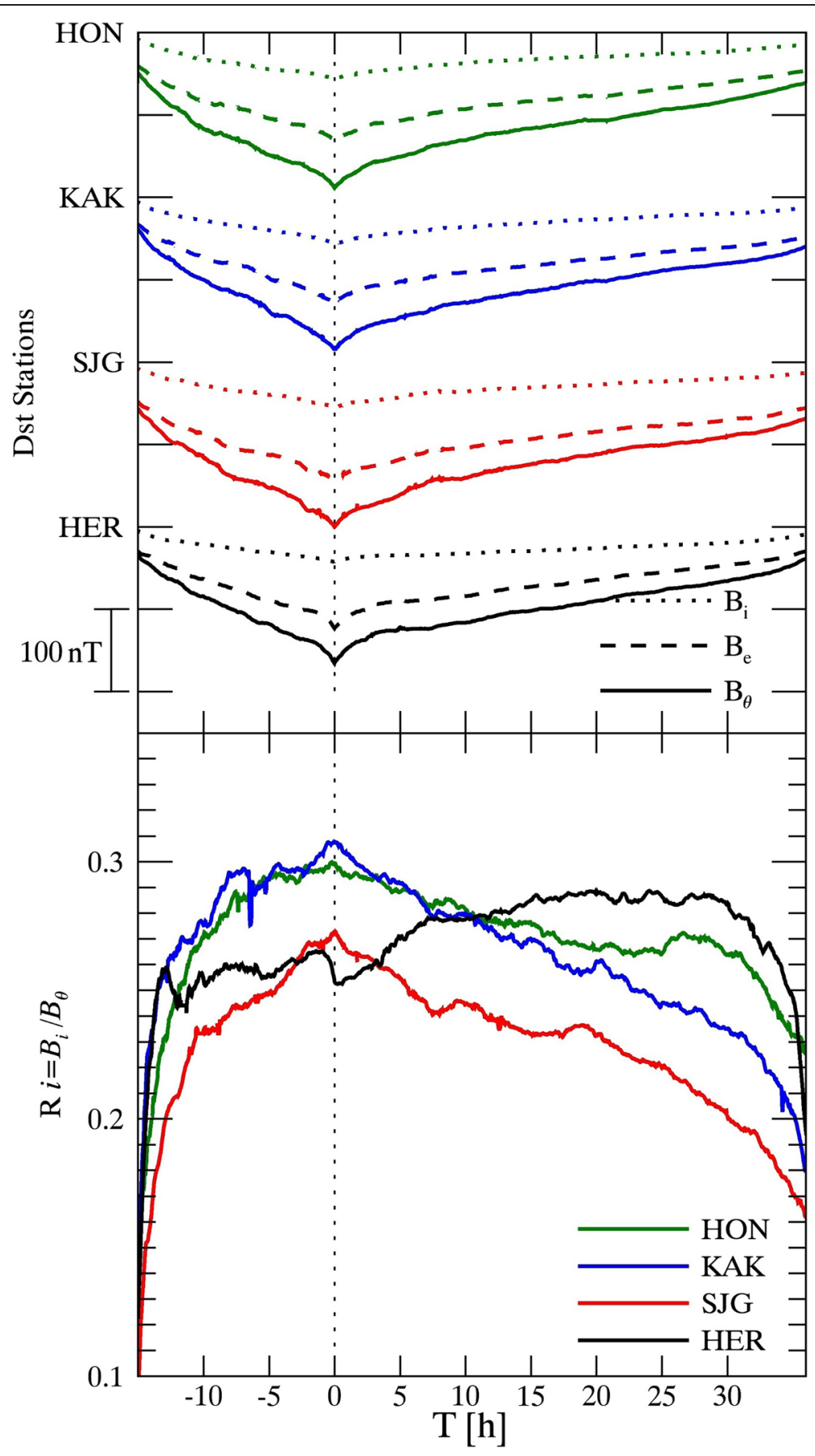

Figure 5 Superposed epoch analysis (SEA) results over all selected storms for each disturbance storm time (Dst) station. The upper panel illustrates the horizontal field $\left(B_{\theta}\right)$ and the internal and external part $\left(B_{i}, B_{e}\right)$ of each station; the lower panel shows the related internal contributions $\left(R_{i}=B_{i} / B_{\theta}\right)$.

upper mantle region and local conductivity differences due to ocean induction created the differences in internal contributions among these Dst stations. With the exception of HER, the top value and entire structure of $R_{i}$ variations in the main and recovery phase regions increased with the intensification of $D s t$ variation or the levels of geomagnetic storms from G1 to G3. It is clearly demonstrated that at
HON, KAK, and SJG, the internal induced magnetic fields have already exceeded the linear growth with respect to the intensification of external source currents.

In equation (3), the widely used Dst index was derived from all four stations. Considering the unusual situation of magnetic ratios at HER station, it was reasonably ruled out in this research. Therefore, although the calculated 
results with equation (3) over HON, KAK, and SJG stations cannot be literally referred to as the Dst index, adopted the same terms for magnetic fields and the ratios just as that used in each station. Figure 6 presents these results, with magnetic components for different storm levels from G1 to G3; the total storms and related ratios are illustrated in the upper and lower panels, respectively.

In Figure 6, the green, blue, and red lines represent G1, G2, and G3, respectively, and black lines represent the averaged value of all selected storms. It is obvious that the internal ratio for the category of total storms, which is represented by the black curve in lower panel, was nearly equal to that of G1. This occurred because the number of events in the G1 category was significantly larger than that in G2 and G3, as shown in Table 1, and naturally carried substantially larger weight in the SEA process.

According to the traces of $R_{i}$ separately plotted in the lower panel of Figure 6, it is obvious that for all storm categories, the internal ratios all logarithmically grow to the top values during the main phase. Moreover, significant

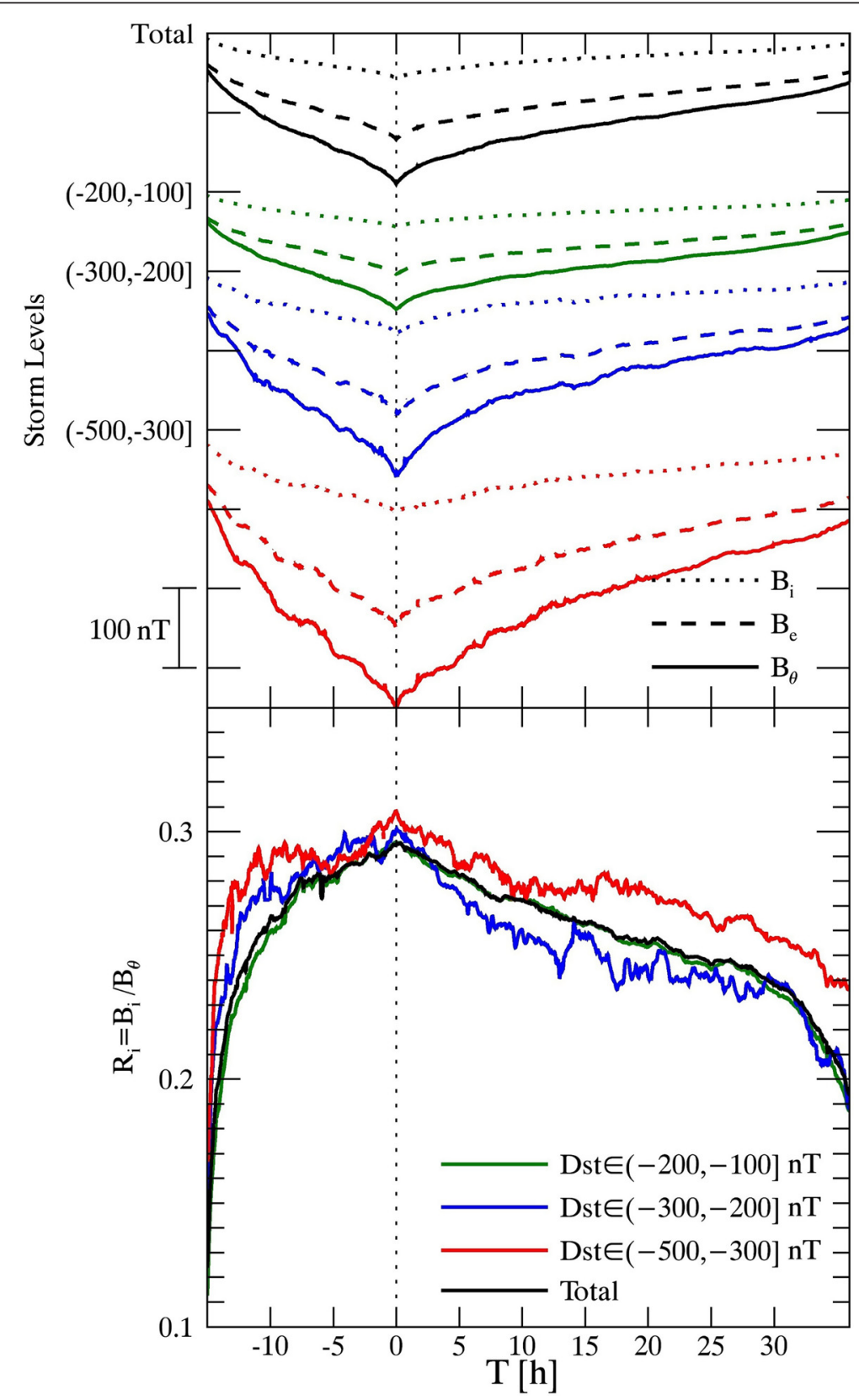

Figure 6 Superposed epoch analysis (SEA) means covering the observations of HON, KAK, and SJG stations for each storm category. The HER station was eliminated for its unusual situation. 
local growths, still in the logarithmic manner, are superimposed on the $R_{i}$ traces for larger storm categories G2 and G3 corresponding to the multiple temporary intensification of the magnetic ring current during the main phase. Consequently, the logarithmic form of the local growth of the internal induced fields actually amplifies these temporary intensifications on surface magnetic observations. Referred to as multistep storms or multiple-dip storms, they obviously appear on the curves of the magnetic components of G2 and G3 in Figure 6 and Figures 1, 2, and 3. The amplification effect to ground magnetic observations is helpful for researchers to more easily identify temporary ring current intensification. Apparently, the corresponding logarithmic growth of the internal induced field to the external field also amplifies the entire storm main phase on ground observations.

At the point of the Dst-minimum, the internal ratios showed a small increase from the G1 category, with ratio values of $0.295 \pm 0.014$, to G2 and G3 categories, holding values of $0.300 \pm 0.016$ and $0.308 \pm 0.015$, respectively, as represented in the lower panel of Figure 6 . This growth is actually legitimate in physics. Since it is based on Faraday's law of induction, $\nabla \times E=-\frac{\partial B}{\partial t}$, the induction of the magnetic field from the internal induced current is linearly related to the partial derivative of the external source field with respect to time rather than to the intensity of the external source field. For the storms with the Dst-minimum less than -200 nT, corresponding to storms in G2 and G3 categories in this study, stronger storms possess shorter main-phase durations on average (Hutchinson et al. 2011) and consequently larger values of the partial derivative of the external source field with respect to time. Therefore, the internal induced magnetic fields on average have larger contributions to surface observations for larger storms, even if the increase of the contribution ratio of the internal field is not dramatic.

\section{Conclusions}

In this paper, we have employed the external source and the internal induced field separation based on the spherical harmonic expansion of the disturbed magnetic field to evaluate the influence of induced currents within the Earth on the surface magnetic variations. The main objective of this study is to provide an understanding of the response performance of geomagnetic induction with different disturbance strengths of symmetric ring current. Our results show that the internal induced field and the external source field both maintain the same variation trend with the typical Dst storm evolution covering the main and recovery phases. At the point of the Dst-minimum, the ratio of internal induced fields to surface observations increases with the intensification of storms, from $0.295 \pm 0.014$ to $0.300 \pm 0.016$ and $0.308 \pm$
0.015 corresponding to storm intensity levels of $G 1$ : $(-200 n T,-100 n T], G 2:(-300 n T,-200 n T]$, and G3: $(-500 n T,-300 n T$ ], respectively. During the storm main phase, the development of internal contribution ratio mostly follows the pattern of logarithmic growth with the evolution of the storm up to the top value at the Dst-minimum before slowly decreasing during the long recovery phase. For larger storms in G2 and G3 categories, remarkable small growths are superimposed on the traces of ratio values during the storm main phase. This effect is caused by the sudden intensification of energetic particle injections into the magnetic ring current and mostly occurs during the periods of the main phases of intense storms. This logarithmic growth pattern of internal induced field obviously amplifies the effects of the ring current intensification on the surface geomagnetic observations and is helpful for researchers to identify the ring current intensification pattern more easily from geomagnetic observations.

It is clear that the geomagnetic induction exceeds linear growth with respect to the increases in external source field, which is physically reasonable and coincident with Faraday's law of induction $\left(\nabla \times E=-\frac{\partial B}{\partial t}\right)$. Since larger storms relate to larger values of the time derivative of the magnetic field under the assumption of lasting the same approximate duration of storm main phases, this growth is actually reasonable for intense storms led by high-speed CMEs. This internal response of the induced field is a compound reaction encompassing the influence of the near-surface environment and the response result of electric conductivity in the upper mantle, which affects the penetration depth of external magnetic signals. Nonetheless, it is still obvious that the contribution rate of the induced field to the surface magnetic field variation increases with an increase in disturbance levels in the magnetospheric ring current.

Although the ratio structure at each station generally follows this pattern, differences are noted among stations. On average, the value of $R_{i}$ at KAK is slightly larger than that at HON but is significantly larger than that at SJG. The tendency of $R_{i}$ variation at HER is different with an unexpected higher value in the recovery phase than that in the main phase. The discrepancy among stations could be explained by the lateral anisotropy of electrical conductivity in the upper region of the Earth's mantle, since the divergence among KAK, HON, and SJG stations in our results is coincident with the lateral anisotropies in conductivity of the upper mantle reported by Kelbert et al. (2009). Moreover, the surface conductivity discrepancy around stations, particularly the effect of high induction in oceans, also contributes to the $R_{i}$ difference among the stations.

The $D s t$ index is widely used and highly valued in space weather prediction and geomagnetic model construction 
to quantify magnetospheric disturbance as a response to interplanetary fluctuation. The research on the observed internal contribution is necessary to provide a comprehensive understanding of Dst index variation. Due to differences in the internal contribution ratio among stations, the internal induced and external source field separation could be an essential procedure for each geomagnetic observatory when using surface geomagnetic observations to research the distribution of magnetospheric current systems.

\section{Abbreviations}

CIR: interplanetary corotating interaction regions; CME: coronal mass ejection; Dst: disturbance storm time; HER: Hermanus; HON: Honolulu; KAK: Kakioka; SEA: superposed epoch analysis; SJG: San Juan; $S_{q}$ : solar quiet daily variation.

\section{Competing interests}

The authors declare that they have no competing interests.

\section{Authors' contributions}

DX developed the main framework. All authors conducted the theoretical considerations and read and approved the final manuscript.

\section{Acknowledgements}

We thank the INTERMAGNET organization for supplying the magnetometer data. This work is supported by The Basic Research and Operational Fund of China Earthquake Administration (DQJB12B25) and the National Science Foundation of China, grant no. 41274079.

Received: 26 July 2014 Accepted: 15 January 2015

Published online: 30 January 2015

\section{References}

Backus G, Parker R, Constable C (1996) Foundations of geomagnetism. Cambridge Univ, Press, New York

Banks RJ (1969) Geomagnetic variations and the electrical conductivity of the upper mantle. Geophys J Roy Astr S 17:457-487, doi:10.1111/j.1365246X.1969.tb00252.x

Constable S (1993) Constraints on mantle electrical conductivity from field and laboratory measurements. J Geomagn Geoelectr 45(9):7070-7728

Constable S, Constable C (2004) Observing geomagnetic induction in magnetic satellite measurements and associated implications for mantle conductivity. Geochem Geophys Geosyst 5, Q01006, doi:10.1029/2003GC000634

Gonzalez W, Joselyn J, Kamide Y, Kroehl H, Rostoker G, Tsurutani B, Vasyliunas V. (1994) What is a geomagnetic storm? J. Geophys. Res.-Space, (1978-2012). doi:10.1029/93JA02867

Gonzalez W, Tsurutani B, Gonzalez A (1999) Interplanetary origin of geomagnetic storms. Space Sci. Rev. doi:10.1023/A:1005160129098

Gonzalez W, Echer E, Tsurutani B, Gonzalez A, Dal Lago A. (2011) Interplanetary origin of intense, superintense and extreme geomagnetic storms. Space Sci. Rev. doi:10.1007/s11214-010-9715-2

Häkkinen LVT, Pulkkinen TI, Nevanlinna H, Pirjola RJ, Tanskanen El. (2002) Effects of induced currents on Dst and on magnetic variations at midlatitude stations, J. Geophys. Res. 107(A1), doi:10.1029/2001JA900130

Hamilton D, Gloeckler G, Ipavich F, Stüdemann W, Wilken B, Kremser G (1988) Ring current development during the great geomagnetic storm of February 1986. J Geophys Res 93:14343-14355, doi:10.1029/JA093iA12p14343

Hutchinson J, Wright D, Milan S (2011) Geomagnetic storms over the last solar cycle: a superposed epoch analysis. J. Geophys. Res., doi:10.1029/ 2011JA016463

Jackson J (1998) Classical electrodynamics. Wiley, New York

Kelbert A, Schultz A, Egbert G (2009) Global electromagnetic induction constraints on transition-zone water content variations. Nature 460 (7258):1003-1006, doi:10.1038/nature08257

Khan A, Kuvshinov A, Semenov A (2011) On the heterogeneous electrical conductivity structure of the Earth's mantle with implications for transition zone water content. J Geophys Res 116:B01103, doi: 10.1029/2010JB007458
Kuvshinov A, Olsen N (2006) A global model of mantle conductivity derived from 5 years of CHAMP, Ørsted, and SAC-C magnetic data. Geophys Res Lett 33:L18301, doi:10.1029/2006GL027083

Liemohn MW, Jazowski M (2008) Ring current simulations of the 90 intense storms during solar cycle 23. J Geophys Res 113:A00A17, doi:10.1029/ 2008JA013466

Lorrain P, Corson D (1970) Electromagnetic fields and waves. WH Freeman, San Francisco

Mareschal M (1986) Modelling of natural sources of magnetospheric origin in the interpretation of regional induction studies: a review. Surv Geophys 8(3):261-300

Martinec Z, McCreadie H (2004) Electromagnetic induction modelling based on satellite magnetic vector data. Geophys J Int 157:10451060, doi:10.1111/ j.1365-246X.2004.02252.x

Olsen N (1999) Long-period (30 days-1 year) electromagnetic sounding and the electrical conductivity of the lower mantle beneath Europe. Geophys J Int 138:179-187, doi:10.1046/j.1365-246x.1999.00854.x

Price AT (1967) Electromagnetic induction within the Earth. In: Matsushita S, Campbell WH (eds) Physics of Geomagnetic Phenomena, vol. I. Academic, San Diego, Calif, p 235

Püthe $P$ (2013a) Determination of the 1-D distribution of electrical conductivity in Earth's mantle from Swarm satellite data. Earth Planets Space 65:12331237, doi:10.5047/eps.2013.07.007

Püthe $P$ (2013b) Determination of the 3-D distribution of electrical conductivity in Earth's mantle from Swarm satellite data: frequency domain approach based on inversion of induced coefficients. Earth Planets Space 65:12471256, doi:10.5047/eps.2013.09.004

Sabaka TJ, Hulot G, Olsen N (2010) Mathematical properties relevant to geomagnetic field modelling. In: Freeden W, Nashed Z, Sonar T (eds) Handbook of Geomathematics. Springer, Heidelberg

Schultz A, Larsen JC (1990) On the electrical conductivity of Earth's interior: II. Delineation of heterogeneity by application of extremal inverse solution. Geophys J Int 101:565-590

Sugiura M (1964) Hourly values of the equatorial Dst for the IGY, Ann. IGY 35:9-45

Sugiura M, Kamei T (1991) Equatorial Dst Index: 1957-1986. In: Berthelier A, Menvielle M (eds) IAGA Bull., vol. 40. ISGI Publ. Off., Saint-Maur-des-Fosses, France

Temerin M, Li X (2002) A new model for the prediction of Dst on the basis of the solar wind. J Geophys Res 107(A12):1472, doi:10.1029/2001JA007532

Temerin M, Li X (2006) Dst model for 1995-2002. J. Geophys. Res., doi:10.1029/ 2005JA011257.

Turner N, Baker D, Pulkkinen T, Roeder J, Fennell J, Jordanova V (2001) Energy content in the storm time ring current. J Geophys Res 106:19149-19156, doi:10.1029/2000JA003025

Velímský V (2013) Determination of three-dimensional distribution of electrical conductivity in the Earth's mantle from Swarm satellite data: time-domain approach. Earth Planets Space 65:12391246, doi:10.5047/eps.2013.08.001

Xu D, Chen T, Zhang X, Liu Z (2009) Statistical relationship between solar wind conditions and geomagnetic storms in 1998-2008. Planet. Space Sci. doi:10.1016/j.pss.2009.07.015

\section{Submit your manuscript to a SpringerOpen ${ }^{\odot}$ journal and benefit from:}

- Convenient online submission

Rigorous peer review

- Immediate publication on acceptance

- Open access: articles freely available online

- High visibility within the field

- Retaining the copyright to your article

Submit your next manuscript at $>$ springeropen.com 